Article

\title{
Short Remarks on Complete Monotonicity of Some Functions
}

\section{Ladislav Matejíčka}

Faculty of Industrial Technologies in Púchov, Trenčín University of Alexander Dubček in Trenčín,

I. Krasku 491/30, 02001 Púchov, Slovakia; ladislav.matejicka@tnuni.sk

Received: 25 February 2020; Accepted: 28 March 2020; Published: 5 April 2020

check for updates

\begin{abstract}
In this paper, we show that the functions $x^{m}\left|\beta^{(m)}(x)\right|$ are not completely monotonic on $(0, \infty)$ for all $m \in \mathbb{N}$, where $\beta(x)$ is the Nielsen's $\beta$-function and we prove the functions $x^{m-1}\left|\beta^{(m)}(x)\right|$ and $x^{m-1}\left|\psi^{(m)}(x)\right|$ are completely monotonic on $(0, \infty)$ for all $m \in \mathbb{N}, m>2$, where $\psi(x)$ denotes the logarithmic derivative of Euler's gamma function .
\end{abstract}

Keywords: completely monotonic functions; laplace transform; inequality; Nielsen's $\beta$-function; polygamma functions

\section{Introduction}

Completely monotonic functions have attracted the attention of many authors. Mathematicians have proved many interesting results on this topic. For example, Koumandos [1] obtained upper and lower polynomial bounds for the function $x /\left(e^{x}-1\right) x>0$, with coefficients of the Bernoulli numbers $B_{k}$. This enabled him to give simpler proofs of some results of H. Alzer and F. Qi et al., concerning complete monotonicity of certain functions involving the functions $\Gamma(x), \psi(x)$ and the polygamma functions $\psi^{(n)}, n=1,2, \ldots$. [2]. For example, he proved by simpler way the following theorem [3].

Theorem 1. The functions

$$
\begin{gathered}
\psi(x)-\log (x)+\frac{1}{2 x}+\frac{1}{12 x^{2}}, \\
\log (x)-\frac{1}{2 x}-\psi(x), \\
\psi^{\prime}(x)-\frac{1}{x}-\frac{1}{2 x^{2}}-\frac{1}{6 x^{3}}+\frac{1}{30 x^{5}}, \\
\frac{1}{x}+\frac{1}{2 x^{2}}+\frac{1}{6 x^{3}}-\psi^{\prime}(x)
\end{gathered}
$$

are strictly completely monotonic on $(0, \infty)$.

Qi and Agarwal [4] surveyed some results related to the function $\left[\psi^{\prime}(x)\right]^{2}+\psi^{\prime \prime}(x)$, its q-analogous, variants, and divided difference forms; several ratios of gamma functions; and so on. Their results include the origins, positivity, inequalities, generalizations, completely monotonic degrees, (logarithmically) complete monotonicity, necessary and sufficient conditions, equivalences to inequalities for sums, applications, etc. Finally, the authors listed several remarks and posed several 
open problems. We note that the seventh open problem ([4] p. 39) was solved by Matejicka [5] and the fourth open problem ([4] p. 38) was solved by Matejicka [6]. H. Alzer et al. [7] disproved the following conjecture:

Conjecture 1. Let $\Phi_{m}(x)=-x^{m} \psi^{(m)}(x)$, where $\psi(x)$ denotes the logarithmic derivative of Euler's gamma function [2]. Then, the function $\Phi_{m}^{(m)}(x)$ is completely monotonic on $(0, \infty)$ for each $m \in \mathbb{N}$.

Clark and Ismail [2] proved that, if $m \in 1,2, \ldots, 16$, then $\Phi_{m}^{(m)}(x)$ is completely monotonic on $(0, \infty)$, and thus they conjectured that this is true for all natural numbers $m$. Alzer, Berg and Koumandos [7] disproved Conjecture 1 by showing that there exists an integer $m_{0}$ such that for all $m \geq m_{0}$ the functions $\Phi_{m}^{(m)}(x)$ are not completely monotonic on $(0, \infty)$. They defined the function

$$
\Delta_{\alpha, m}(x)=x^{\alpha}\left|\psi^{(m)}(x)\right| \text { for } x>0, m \in \mathbb{N}, \alpha \in \mathbb{R},
$$

and noted that it remains an open problem to determine all $(\alpha, m) \in \mathbb{R}^{+} \times \mathbb{N}$ such that $\Delta_{\alpha, m}$ is completely monotonic. We note that this problem is still open.

Nantomah ([8] p. 92) posed a similar open problem: Find all values of $a \in \mathbb{R}$ such that the function $H_{a}(x)=x^{a}\left|\beta^{(m)}(x)\right|$ is completely monotonic on $(0, \infty)$, where $\beta(x)$ is the Nielsen's $\beta$-function.

Alzer, Berg and Koumandos [7] introduced new function

$$
s(x)=\frac{1}{2}+\frac{1}{\pi} H\left(\frac{x}{2 \pi}\right)
$$

where

$$
H(x)=\sum_{k=1}^{\infty} \frac{1}{k} \sin \left(\frac{x}{k}\right)
$$

is the Hardy-Littlewood function [9-13] defined for $x \in \mathbb{C}$. They showed that the functions $\Phi_{m}^{(m)}(x)$ are all completely monotonic on $(0, \infty), m \in \mathbb{N}$ if and only if $s(x) \geq 0$ for $x>0$. Their main result was that for each $K>0$ there is $x_{K}>0$ such that $H\left(x_{K}\right)<-K$. It implies Conjecture 1 is not valid.

The goal of this paper is to show that the functions $Y_{m}(x)=x^{m}\left|\beta^{(m)}(x)\right|$ are not completely monotonic on $(0, \infty)$ for all $m \in \mathbb{N}$, but the functions $x^{m-1}\left|\psi^{(m)}(x)\right|, x^{m-1}\left|\beta^{(m)}(x)\right|$ are completely monotonic on $(0, \infty)$ for all $m \in \mathbb{N}, m>2$. A detailed list of references on completely monotonic functions can be found in [1-39].

Now, recall some useful definitions and theorems. The classical Nielsen's $\beta$-function $[8,31,32]$ is defined as

\section{Definition 1.}

$$
\begin{aligned}
\beta(x) & =\int_{0}^{\infty} \frac{e^{-x t}}{1+e^{-t}} d t=\int_{0}^{1} \frac{t^{x-1}}{1+t} d t=\sum_{m=0}^{\infty} \frac{(-1)^{m}}{m+x}= \\
& =\frac{1}{2}\left(\psi\left(\frac{1+x}{2}\right)-\psi\left(\frac{x}{2}\right)\right)
\end{aligned}
$$

for $x>0$, where $\psi(x)=d \ln \Gamma(x) / d x$ is a digamma function and $\Gamma(x)$ is the Euler's Gamma function [4,24]. 
It is generally known (see [8]) that the special function $\beta(x)$ is related to the Euler's beta function $B(x, y)$ and to the Gauss hypergeometric function ${ }_{2} F_{1}(a, b ; c ; d)$ by

$$
\begin{aligned}
& \beta(x)=-\frac{d}{d x}\left(\ln B\left(\frac{x}{2}, \frac{1}{2}\right)\right) \\
& \beta(x)=\frac{1}{x}\left(2 F_{1}(1, x ; 1+x ;-1)\right)
\end{aligned}
$$

for $x>0$.

For additional information on the Nielsen's $\beta$-function, one may refer to [8,32] and the related references therein.

Definition $2([4,24])$. We say that a function $f$ is a completely monotonic on the interval I, if $f(x)$ has derivatives of all orders on I and the inequality $(-1)^{n} f^{(n)}(x) \geq 0$ holds for $x \in I$ and $n \in \mathbb{N}_{0}$.

A characterization of completely monotonic function can be given by the Bernsten-Widder theorem [36,37], which reads that a function $f(x)$ on $(0, \infty)$ is completely monotonic if and only if there exists bounded and non-decreasing function $\alpha(t)$ such that the integral

$$
f(x)=\int_{0}^{\infty} e^{-x t} d \alpha(t)
$$

converges for $x \in(0, \infty)$.

Definition 3 ([24]). Let $h(t)$ be a completely monotonic function on $(0, \infty)$ and let $h(\infty)=\lim _{t \rightarrow \infty} h(t) \geq 0$. If the function $t^{\alpha}[h(t)-h(\infty)]$ is a completely monotonic on $(0, \infty)$ when and only when $0 \leq \alpha \leq r \in \mathbb{R}$, then we say $h(t)$ is of completely monotonic degree $r$; if $t^{\alpha}[h(t)-h(\infty)]$ is a completely monotonic on $(0, \infty)$ for all $\alpha \in \mathbb{R}$, then we say that the completely monotonic degree of $h(t)$ is $\infty$.

For convenience, Guo [21] designed a notation $\operatorname{deg}_{\mathrm{cm}}^{t} h(t)$ to denote the completely monotonic degree $r$ of $h(t)$ with respect to $t \in(0, \infty)$.

Matejicka [31] s showed that the function $x^{m}\left|\beta^{(m)}(x)\right|$ is completely monotonic on $(0, \infty)$ for $m=1,2,3$.

Definition 4 ([35]). A function $f$ has exponential order $\alpha$ if there exist constants $M>0$ and $\alpha$ such that for some $t_{0} \geq 0$

$$
|f(t)| \leq M e^{\alpha t}, t \geq t_{0}
$$

Definition 5. We say

$$
\alpha^{*}=\inf \left\{\alpha ; \text { there are constants } M, t_{0} \text { such that }|f(t)| \leq M e^{\alpha t}, t \geq t_{0}\right\}
$$

is a lower exponential order of function $f$.

Theorem $2([19,20])$. (Weierstrass's criterion for uniform convergence) Suppose $f(x, t)$ is a continuous on $[a, b] \times[0, \infty)$ and $g(t)$ is integrable on $[0, \infty)$. If $|f(x, t)| \leq g(t)$ for all $a \leq x \leq b$ and all $t \geq 0$ then the integral $\int_{0}^{\infty} f(x, t) d t$ is uniformly convergent on $[a, b]$. 
Theorem 3 ([19,20]). Suppose $f(x, t)$ is continuous on $[a, b] \times[0, \infty)$ together with its partial derivative $\frac{\partial f}{\partial x}(x, t)$. In this case,

$$
\frac{d}{d x} \int_{0}^{\infty} f(x, t) d t=\int_{0}^{\infty} \frac{\partial}{\partial x} f(x, t) d t
$$

when the first integral is convergent and the second is uniformly convergent on $[a, b]$.

\section{Main Results}

Theorem 4. Let $m \in \mathbb{N}$ and $\varphi, \varphi^{\prime}, \ldots \varphi^{(m)}$ be continuous functions of lower exponential orders $L_{0}^{*}, L_{1}^{*}, \ldots L_{m \prime \prime}^{*}$ respectively, on $(0, \infty)$. Let

$$
\begin{gathered}
L=\max \left\{L_{0}^{*}, L_{1}^{*}, \ldots, L_{m}^{*}\right\} \geq 0 ; F(x)=\int_{0}^{\infty} \varphi(t) e^{-x t} d t \geq 0 \text { for } x>L ; \varphi^{(m)}(t) \geq 0 \text { on }(0, \infty) . \text { Let } \\
p_{1}: \quad \lim _{t \rightarrow+\infty} \varphi^{(k)}(t) \frac{\partial^{m-1-k}}{\partial t^{m-1-k}}\left(t^{n} e^{-x t}\right)=0
\end{gathered}
$$

and

$$
p_{2}: \quad \lim _{t \rightarrow 0^{+}} \varphi^{(k)}(t) \frac{\partial^{m-1-k}}{\partial t^{m-1-k}}\left(t^{n} e^{-x t}\right)=0
$$

for $x>L, n \in \mathbb{N}, k=0, \ldots, m-1$. Then, $x^{m} F(x)$ is a completely monotonic function on $(L,+\infty)$.

Remark 1. We note that, if the following conditions

$$
\lim _{t \rightarrow 0^{+}} \varphi^{(k)}(t)=0, \quad \lim _{t \rightarrow \infty} \varphi^{(k)}(t) e^{-x t}=0
$$

are fulfilled for $k=0, \ldots, m-1$, and $x>L$ where $m \in \mathbb{N}$, then $p_{1}, p_{2}$ are also valid.

Proof. Let $a, b$ be fixed and such that $L<a<b$. To prove Theorem 4 , it suffices to show

$$
L_{m, n}=(-1)^{n}\left(x^{m} F(x)\right)^{(n)} \geq 0
$$

for $n \geq 1$ and $a \leq x \leq b$. Some computation gives

$$
L_{m, n}=(-1)^{n+m}\left(\int_{0}^{\infty} \varphi(t) \frac{\partial^{m}}{\partial t^{m}} e^{-x t} d t\right)^{(n)}
$$

Denote

$$
f(x, t)=\varphi(t) x^{m} e^{-x t}
$$

Let $\varepsilon^{*}>0$ such that $\varepsilon^{*}<a-L$. Then, there are $M, t_{0}$ such that $|\varphi(t)| \leq M e^{\left(L+\varepsilon^{*}\right) t}$ for $t \geq t_{0}$. This implies

$$
|f(x, t)| \leq M e^{\left(L+\varepsilon^{*}\right) t}(\max \{|a|,|b|\})^{m} e^{-x t} \leq g(t)=C(a, b, m, M) e^{\left(L+\varepsilon^{*}-a\right) t} \text { for } t \geq t_{0} .
$$

It is evident that $g(t)$ is an integrable function on $(0,+\infty)$.

A straightforward differentiation yields

$$
\frac{\partial}{\partial x} f(x, t)=\varphi(t) m x^{m-1} e^{-x t}-t \varphi(t) x^{m} e^{-x t} .
$$


It follows immediately that

$$
\left|\frac{\partial}{\partial x} f(x, t)\right| \leq C(a, b, m, M) e^{\left(L+\varepsilon^{*}-a\right) t}\left(\frac{m}{a}+t\right) \text { for } t \geq t_{0} .
$$

Thus, according to Theorems 2 and 3, the integral

$$
\int_{0}^{\infty} \frac{\partial}{\partial x} f(x, t) d t
$$

is uniformly convergent on $[a, b]$ and we observe that

$$
\frac{d}{d x}\left(\int_{0}^{\infty} f(x, t) d t\right)=\int_{0}^{\infty} \frac{\partial}{\partial x} f(x, t) d t
$$

It is easy to see that the function $h(t)=t^{k} e^{-\varepsilon t}$, where $k \in \mathbb{N}, \varepsilon=a-L-\varepsilon^{*}$ is integrable on $(0,+\infty)$. Using mathematical induction and considering the formulas

$$
\int_{0}^{\infty} t e^{-\varepsilon t} d t=\frac{1}{\varepsilon^{2}}, \int_{0}^{\infty} t^{k} e^{-\varepsilon t} d t=\frac{k}{\varepsilon} \int_{0}^{\infty} t^{k-1} e^{-\varepsilon t} d t
$$

for $k \in \mathbb{N}, \varepsilon>0$ bring the desired result. If we repeat the process $\mathrm{n}$ times, we obtain

$$
L_{m, n}=(-1)^{n+m} \int_{0}^{\infty} \varphi(t) \frac{\partial^{n}}{\partial x^{n}}\left(\frac{\partial^{m}}{\partial t^{m}} e^{-x t} d t\right)
$$

Using Schwartz theorem (mixed partial derivatives) [19] yields

$$
L_{m, n}=(-1)^{n+m} \int_{0}^{\infty} \varphi(t) \frac{\partial^{m}}{\partial t^{m}}\left(\frac{\partial^{n}}{\partial x^{n}} e^{-x t} d t\right)=(-1)^{m} \int_{0}^{\infty} \varphi(t) \frac{\partial^{m}}{\partial t^{m}}\left(t^{n} e^{-x t} d t\right) d t
$$

Applying to $L_{m, n}$ integration by parts $m$ times and using $p_{1}, p_{2}$ leads to

$$
L_{m, n}=\int_{0}^{\infty} \varphi^{(m)}(t) t^{n} e^{-x t} d t
$$

This completes the proof of our theorem.

Lemma 1. Let

$$
\varphi_{1}(t)=\frac{t^{m}}{1-e^{-t}} \quad \text { and } \quad \varphi_{2}(t)=\frac{t^{m}}{1+e^{-t}}
$$

for $t>0$ and $m>2, m \in \mathbb{N}$. Then,

(1) $\lim _{t \rightarrow a} \varphi_{2}^{(k)}(t) \frac{\partial^{m-1-k}}{\partial t^{m-1-k}}\left(t^{n} e^{-x t}\right)=0$ for $a=0^{+}$and $a=+\infty, k=0, \ldots, m-1, n \in \mathbb{N}, x>0$,

(2) $\lim _{t \rightarrow a} \varphi_{i}^{(k)}(t) \frac{\partial^{m-2-k}}{\partial t^{m-2-k}}\left(t^{n} e^{-x t}\right)=0$ for $i=1,2, a=0^{+}$and $a=+\infty, k=0, \ldots, m-2, n \in \mathbb{N}, x>0$.

Proof. Differentiating $k$ times the following equations

$$
\left(1-e^{-t}\right) \varphi_{1}(t)=t^{m} \quad \text { and } \quad\left(1+e^{-t}\right) \varphi_{2}(t)=t^{m}
$$


leads to

$$
\varphi_{1}^{(k)}(t)=\left[\left(t^{m}\right)^{(k)}+\sum_{j=0}^{k-1}\left(\begin{array}{c}
k \\
j
\end{array}\right)(-1)^{k-j} \varphi_{1}^{(j)}(t) e^{-t}\right] \frac{1}{1-e^{-t}}
$$

and

$$
\varphi_{2}^{(k)}(t)=\left[\left(t^{m}\right)^{(k)}-\sum_{j=0}^{k-1}\left(\begin{array}{c}
k \\
j
\end{array}\right)(-1)^{k-j} \varphi_{2}^{(j)}(t) e^{-t}\right] \frac{1}{1+e^{-t}} .
$$

Similarly, we get

$$
\alpha=\frac{\partial^{m-1-k}}{\partial t^{m-1-k}}\left(t^{n} e^{-x t}\right)=e^{-x t} \sum_{i=0}^{m-1-k}\left(\begin{array}{c}
m-1-k \\
i
\end{array}\right)(-1)^{m-1-k-i}\left(t^{n}\right)^{(i)} x^{m-1-k-i}
$$

and

$$
\gamma=\frac{\partial^{m-2-k}}{\partial t^{m-2-k}}\left(t^{n} e^{-x t}\right)=e^{-x t} \sum_{i=0}^{m-2-k}\left(\begin{array}{c}
m-2-k \\
i
\end{array}\right)(-1)^{m-2-k-i}\left(t^{n}\right)^{(i)} x^{m-2-k-i}
$$

H. Alzer et al. [7] (p. 108) demonstrated that the generating function for Bernoulli numbers $B_{k}$ yields for $|t|<2 \pi$

$$
\frac{t}{1-e^{-t}}=1+\frac{t}{2}+\sum_{k=2}^{\infty} \frac{B_{k}}{k !} t^{k}
$$

the series on the right side of Equation (5) is uniformly convergent on each $[-c, c]$ where $0<c<2 \pi$. This implies

$$
\varphi_{1}(t)=t^{m-1}+\frac{t^{m}}{2}+\sum_{j=2}^{\infty} \frac{B_{j}}{j !} t^{j+m-1}
$$

and

$$
\varphi_{1}^{(k)}(t)=\left(t^{m-1}\right)^{(k)}+\frac{\left(t^{m}\right)^{(k)}}{2}+\sum_{j=2}^{\infty} \frac{B_{j}}{j !}\left(t^{j+m-1}\right)^{(k)} .
$$

From Equation (6), we get $\lim _{t \rightarrow 0^{+}} \varphi_{1}^{(k)}(t)=0$ for $k=0, \ldots, m-2$. Using mathematical induction and Equation (2), we deduce that $\lim _{t \rightarrow 0^{+}} \varphi_{2}^{(k)}(t)=0$ for $k=0, \ldots, m-1$. It is evident that $\lim _{t \rightarrow 0^{+}} \alpha=0$ or $\lim _{t \rightarrow 0^{+}} \alpha=c$ where $c \in \mathbb{R}$. Thus, if $a=0$, then (1) is valid. Next, we have $\left.\alpha^{*}=\varphi_{2}^{(k)}(t) \frac{\partial^{m-1-k}}{\partial t^{m-1-k}}\left(t^{n} e^{-x t}\right)=\varphi_{2}^{(k)}(t) e^{-\frac{x}{2} t} e^{-\frac{x}{2} t^{m-1-k}} \sum_{i=0}^{m-1-k} \begin{array}{c}m-1 \\ i\end{array}\right)(-1)^{m-1-k-i}\left(t^{n}\right)^{(i)} x^{m-1-k-i}$.

By using mathematical induction it is easy to show that $\lim _{t \rightarrow \infty} \varphi_{2}^{(k)}(t) e^{-\frac{x}{2} t}=0$ for $k=0, \ldots, m-1$. Thus, $\lim _{t \rightarrow \infty} \alpha^{*}=0$ for $k=0, \ldots, m-1$. The proof of (1) is complete. 
Similarly, it can be shown that

$$
\lim _{t \rightarrow \infty} \varphi_{1}^{(k)}(t) \frac{\partial^{m-2-k}}{\partial t^{m-2-k}}\left(t^{n} e^{-x t}\right)=0
$$

for $k=0, \ldots, m-2$ and

$$
\lim _{t \rightarrow \infty} \varphi_{2}^{(k)}(t) \frac{\partial^{m-2-k}}{\partial t^{m-2-k}}\left(t^{n} e^{-x t}\right)=0 .
$$

The proof of the Lemma 1 would be done if we show

$$
\lim _{t \rightarrow 0^{+}} \varphi_{1}^{(k)}(t) \frac{\partial^{m-2-k}}{\partial t^{m-2-k}}\left(t^{n} e^{-x t}\right)=0
$$

for $k=0, \ldots, m-2, n \in \mathbb{N}, x>0$. However, it follows from Equation (6). This completes our proof.

Theorem 5. There exists an integer $m_{0}$ such that for each $m>m_{0}$ the function $x^{m}\left|\beta^{(m)}(x)\right|$ is not completely monotonic on $(0, \infty)$.

Proof. Using Theorem 4 and Lemma 1 yields

$$
L_{m, n}=(-1)^{n}\left(x^{m}\left|\beta^{(m)}(x)\right|\right)^{(n)}=\int_{0}^{\infty} \frac{d^{m}}{d t^{m}}\left(\frac{t^{m}}{1+e^{-t}}\right) t^{n} e^{-x t} d t .
$$

Denote

$$
f_{m}(t)=\frac{d^{m}}{d t^{m}}\left(\frac{t^{m}}{1-e^{-t}}\right) \quad \text { and } \quad g_{m}(t)=\frac{d^{m}}{d t^{m}}\left(\frac{t^{m}}{1+e^{-t}}\right) .
$$

H. Alzer et al. [7] (p. 109 (3.4)) proved that

$$
\lim _{m \rightarrow \infty} \frac{1}{m !} f_{m}\left(\frac{x}{m}\right)=s(x) \text { for } \quad x>0,
$$

where ([7] p. 109 (3.7))

$$
s(x)=\frac{1}{2}+\frac{1}{\pi} H\left(\frac{x}{2 \pi}\right)
$$

and

$$
H(x)=\sum_{k=1}^{\infty} \frac{1}{k} \sin \left(\frac{x}{k}\right) \quad \text { for } \quad x \in \mathbb{C} .
$$

Elementary calculation yields

$$
\frac{1}{1+e^{-t}}+\frac{1}{1-e^{-t}}=\frac{2}{1-e^{-2 t}}
$$

A straightforward computation gives

$$
\frac{d^{m}}{d t^{m}}\left(\frac{t^{m}}{1+e^{-t}}\right)+\frac{d^{m}}{d t^{m}}\left(\frac{t^{m}}{1-e^{-t}}\right)=\frac{1}{2^{m-1}} \frac{d^{m}}{d t^{m}}\left(\frac{(2 t)^{m}}{1-e^{-2 t}}\right)
$$


This establishes

$$
g_{m}(x)=2 f_{m}(2 x)-f_{m}(x) .
$$

By using (see ([7] p. 109 (3.4)))

$$
\lim _{m \rightarrow \infty} \frac{f_{m}(x)}{m !}=s(x)
$$

we obtain

$$
g(x)=\lim _{m \rightarrow \infty} \frac{g_{m}(x)}{m !}=2 s(2 x)-s(x) .
$$

Let $\mathrm{m}$ be any natural number and $x^{m}\left|\beta^{(m)}(x)\right|$ be completely monotonic on $(0,+\infty)$. The Bernstein-Widder theorem [36] implies $g_{m}(x) \geq 0$ on $(0,+\infty)$. Thus, if $x^{m}\left|\beta^{(m)}(x)\right|$ is completely monotonic on $(0,+\infty)$ for all $m \in \mathbb{N}$ then $g(x) \geq 0$ on $(0,+\infty)$. It is easy to see that $s(0)=1 / 2$. Continuity of $s(x)$ at 0 gives that there is $\varepsilon>0$ such that $s(x)>0$ for $x \in(0, \varepsilon)$. From Theorem 2.1 (see ([7] p. 105)), we can derive that there is $x_{0}>0$ such that $s\left(x_{0}\right)<0$. Denote

$$
M=\left\{x_{0} ; \text { such that } s\left(x_{0}\right)=0\right\} .
$$

It is obvious that $M \neq \varnothing$. Then, there is $x^{*}=\inf M$. It is easy to see that $0<\varepsilon \leq x^{*}$ and $s\left(x^{*}\right)=0$. From the definition of $x^{*}$, we deduce

$$
g\left(\frac{x^{*}}{2}\right)=2 s\left(x^{*}\right)-s\left(\frac{x^{*}}{2}\right)=-s\left(\frac{x^{*}}{2}\right)<0 .
$$

Thus, there is $m_{0} \in \mathbb{N}$ such that, if $m \geq m_{0}, m \in \mathbb{N}$, then $g_{m}\left(x^{*} / 2\right)<0$. The Bernstein-Widder theorem [36] implies $x^{m}\left|\beta^{(m)}(x)\right|$ is not completely monotonic on $(0, \infty)$. This completes the proof.

Lemma 2. Let $y>0$. Then,

$$
V(y)=\sum_{n=1}^{\infty} \sin ^{2}\left(\frac{y}{n}\right)-\sum_{n=1}^{\infty} \sin ^{2}\left(\frac{y}{2 n}\right)>0 .
$$

Proof. Straightforward calculation gives that, for $m \in \mathbb{N}$,

$$
V(y)=A_{m}(y)-B_{m}(y)+C_{m}(y)
$$

where

$$
\begin{aligned}
& A_{m}(y)=\sum_{n=1}^{m+1} \sin ^{2}\left(\frac{y}{2 n-1}\right), \\
& B_{m}(y)=\sum_{n=1}^{m+1} \sin ^{2}\left(\frac{y}{2 m+2 n}\right)
\end{aligned}
$$


and

$$
C_{m}=\sum_{n=2 m+2}^{\infty}\left(\sin ^{2}\left(\frac{y}{n}\right)-\sin ^{2}\left(\frac{y}{2 n}\right)\right)
$$

It is clear that, if $0<y<\pi(2 m+2) / 2$, then $\sin ^{2}(y /(2 m+2))<y^{2} /(2 m+2)^{2}$. Thus, we obtain that

$$
-B_{m}(y)>-y^{2}\left(\sum_{n=1}^{m+1} \frac{1}{(2 m+2 n)^{2}}\right) .
$$

There are two cases:

- $\quad(\alpha) 0<y \neq k \pi$ for all $k \in \mathbb{N}$.

- $\quad(\beta) y=k_{0} \pi$ for some $k_{0} \in \mathbb{N}$.

In the case $(\alpha)$, there is $j_{0} \in \mathbb{N}$ such that $y<\pi\left(2 j_{0}+2\right) / 2$. Put $\epsilon=\sin ^{2}(y)>0$. It is obvious that there is $m_{0} \in \mathbb{N}, m_{0}>j_{0}$ such that $\left|C_{m}(y)\right|<\epsilon / 2$ and $B_{m}(y)<\epsilon / 2$ for $m>m_{0}$. This implies $V(y)>0$.

Consider the case $\beta$ ) $y=k_{0} \pi$ for some $k_{0} \in \mathbb{N}$. One can easily see that $\epsilon=\sin ^{2}\left(k_{0} \pi /\left(2 k_{0}+1\right)\right)>$ 0 . It is obvious again that there is $m_{0}>k_{0}$ such that $\left|C_{m}(y)\right|<\epsilon / 2$ and $B_{m}(y)<\epsilon / 2$ for $m>m_{0}$. This implies $V(y)>0$. The proof is complete.

Theorem 6. Let $m \in \mathbb{N}, m>2$. Then, $x^{m-1}\left|\psi^{(m)}(x)\right|$ is completely monotonic function on $(0,+\infty)$.

Proof. We need to prove

$$
K_{m, n}=(-1)^{n}\left(x^{m-1}\left|\psi^{(m)}(x)\right|\right)^{(n)} \geq 0
$$

for $x>0, n \in \mathbb{N}$.

Theorem 4 and Lemma 1 imply that the proof will be done if we show

$$
r_{m}(t)=\left(\frac{t^{m}}{1-e^{-t}}\right)^{(m-1)} \geq 0
$$

for $t>0$ and $m \in \mathbb{N}, m>2$. It is clear that

$$
r_{m}(t)=\int_{0}^{t} f_{m}(u) d u+r_{m}(0),
$$

where

$$
f_{m}(t)=\left(\frac{t^{m}}{1-e^{-t}}\right)^{(m)}
$$

The formula ([7] p. 108)

$$
\frac{x^{m}}{1-e^{-x}}=x^{m-1}+\frac{x^{m}}{2}+\sum_{k=2}^{\infty} \frac{B_{k}}{k !} x^{k+m-1}
$$


for $0<x<2 \pi$, where $B_{k}$ are Bernoulli numbers, implies $r_{m}(0)=(m-1)$ !. The proof would be done if we prove $r_{m}(x) \geq 0$ for $x>0$. H. Alzer et al. [7] (p.113) showed that

$$
f_{m}(x)=\int_{0}^{\infty} e^{-t} t^{m} s(x t) d t
$$

for $|x|<2 \pi$, where

$$
s(y)=\frac{1}{2}+\frac{1}{\pi} H\left(\frac{y}{2 \pi}\right)
$$

and

$$
H(y)=\sum_{n=1}^{\infty} \frac{1}{n} \sin \left(\frac{y}{n}\right)
$$

This implies

$$
r_{m}(u)=\int_{0}^{u} \int_{0}^{\infty} e^{-t} t^{m} s(v t) d t d v+(m-1) !
$$

for $|u|<2 \pi$ which can be rewritten as

$$
r_{m}(u)=\int_{0}^{\infty} e^{-t} t^{m}\left(\int_{0}^{u} s(v t) d v\right) d t+(m-1) !
$$

Direct computation yields

$$
\int_{0}^{u} s(v t) d v=\frac{1}{2} u+\frac{1}{\pi} \int_{0}^{u} H\left(\frac{v t}{2 \pi}\right) d v=\frac{1}{2} u+\frac{2}{t} \int_{0}^{\frac{u t}{2 \pi}} H(p) d p>0
$$

because of (see ([13] p. 1))

$$
\int_{0}^{x^{*}} H(p) d p=2 \sum_{n=1}^{\infty} \sin ^{2}\left(\frac{x^{*}}{2 n}\right) \geq 0 .
$$

Thus, $r_{m}(u)>0$ for $0<|u|<2 \pi$.

H. Alzer et al. [7] (p. 112) showed that $f_{m}(x) \geq 0$ for $x \geq 2 \log 2, m \in \mathbb{N}$. Thus, if $x \geq 2 \pi$, then

$$
\begin{aligned}
r_{m}(x) & =\int_{0}^{x} f_{m}(p) d p+(m-1) !=\int_{0}^{2 \log 2} f_{m}(p) d p+\int_{2 \log 2}^{x} f_{m}(p) d p+(m-1) ! \\
& =r_{m}(2 \log 2)+\int_{2 \log 2}^{x} f_{m}(p) d p \geq 0 .
\end{aligned}
$$

This completes the proof.

Theorem 7. Let $m \in \mathbb{N}, m>2$. Then, $x^{m-1}\left|\beta^{(m)}(x)\right|$ is completely monotonic function on $(0,+\infty)$. 
Proof. We need to show

$$
J_{m, n}=(-1)^{n}\left(x^{m-1}\left|\beta^{(m)}(x)\right|\right)^{(n)} \geq 0
$$

for $n \in \mathbb{N}, x>0$. Theorem 4 and Lemma 1 imply that the proof would be done if we show

$$
h_{m}(t)=\left(\frac{t^{m}}{1+e^{-t}}\right)^{(m-1)} \geq 0 .
$$

It is clear that

$$
h_{m}(x)=\int_{0}^{x} g_{m}(t) d t+h_{m}(0) .
$$

From Equation (8), we get

$$
\frac{d^{m-1}}{d t^{m-1}}\left(\frac{t^{m}}{1+e^{-t}}\right)+\frac{d^{m-1}}{d t^{m-1}}\left(\frac{t^{m}}{1-e^{-t}}\right)=\frac{1}{2^{m-1}} \frac{d^{m-1}}{d t^{m-1}}\left(\frac{(2 t)^{m}}{1-e^{-2 t}}\right)
$$

which can be rewritten as $h_{m}(t)=r_{m}(2 t)-r_{m}(t)$. This implies $h_{m}(0)=0$. In the previous part, Equation (10)

$$
g_{m}(x)=2 f_{m}(2 x)-f_{m}(x)
$$

is derived. As a direct consequence, we deduce

$$
h_{m}(x)=\int_{0}^{x} 2 f_{m}(2 t)-f_{m}(t) d t .
$$

Let $0<x<\pi$. Due to result ([7] p. 113)

$$
f_{m}(x)=\int_{0}^{\infty} e^{-t} t^{m} s(x t) d t \quad \text { for } \quad|x|<2 \pi,
$$

we obtain

$$
\begin{aligned}
h_{m}(x) & =\int_{0}^{x}\left(2 \int_{0}^{\infty} e^{-t} t^{m} s(2 u t) d t-\int_{0}^{\infty} e^{-t} t^{m} s(u t) d t\right) d u \\
& =\int_{0}^{\infty} e^{-t} t^{m}\left(\int_{0}^{2 x} s(v t) d v-\int_{0}^{x} s(v t) d v\right) d t=\int_{0}^{\infty} e^{-t} t^{m}\left(\int_{x}^{2 x} s(v t) d v\right) d t .
\end{aligned}
$$

To prove our theorem, it suffices to show that

$$
\int_{x}^{2 x} s(v t) d v \geq 0
$$


for $0<x<\pi$ and $t>0$. Simple computation yields

$$
\int_{x}^{2 x} s(v t) d v=\int_{x}^{2 x} \frac{1}{2}+\frac{1}{\pi} H\left(\frac{v t}{2 \pi}\right) d v=\frac{x}{2}+\frac{2}{t} \int_{\frac{x t}{2 \pi}}^{\frac{x t}{\pi}} H(u) d u .
$$

The proof would be done if we show

$$
\int_{0}^{2 y} H(v) d v-\int_{0}^{y} H(v) d v \geq 0 .
$$

However, it follows from Segal [13] that

$$
\int_{0}^{y} H(v) d v=2 \sum_{n=1}^{\infty} \sin ^{2}\left(\frac{y}{2 n}\right)
$$

and Lemma 2.

Let $x \geq 2 \log 2$. One can easily determine

$$
h_{m}(x)=\int_{0}^{x} g_{m}(t) d t=\int_{0}^{x} 2 f_{m}(2 t)-f_{m}(t) d t=\int_{x}^{2 x} f_{m}(t) d t .
$$

H. Alzer et al. [7] (p. 112) derived that $f_{m}(x) \geq 0$ for $x \geq 2 \log 2$. Thus, $h_{m}(x) \geq 0$ for $x \geq 2 \log 2$. This implies the proof of our theorem.

Remark 2. We note that, in $[8,31]$, it was proved that the function

$$
x^{m}\left|\beta^{(m)}(x)\right|
$$

is strictly completely monotonic on $(0, \infty)$ for $m=2,3$, respectively, for $m=1$.

Remark 3. We note that it is easy to show that functions

$$
x^{m}\left|\beta^{(m)}(x)\right|
$$

are strictly completely monotonic on $(0, \infty)$ for $m=4,5,6$ by using Theorem 4 .

Remark 4. We note that our results and results obtained in [7] imply that

$$
m-1 \leq \operatorname{deg}_{c m}^{t}\left|\psi^{(m)}(t)\right| \leq m
$$

and

$$
m-1 \leq \operatorname{deg}_{c m}^{t}\left|\beta^{(m)}(t)\right| \leq m+1
$$

for $m \in \mathbb{N}, m \geq 3$.

\section{Open Problem}

It is natural to pose the following problem. 
1. Find the value $m_{\beta}$ such that $x^{m}\left|\beta^{(m)}(x)\right|$ is not completely monotonic on $(0,+\infty)$ for $m \geq m_{\beta}$, and $m_{\beta}=\min \left\{m_{0} ; x^{m}\left|\beta^{(m)}(x)\right|\right.$ is not completely monotonic on $(0,+\infty)$ for $\left.m \geq m_{0}\right\}$.

2. Find the value $m_{\psi}$ such that $x^{m}\left|\psi^{(m)}(x)\right|$ is not completely monotonic on $(0,+\infty)$ for $m \geq m_{\psi}$, and $m_{\psi}=\min \left\{m_{1} ; x^{m}\left|\psi^{(m)}(x)\right|\right.$ is not completely monotonic on $(0,+\infty)$ for $\left.m \geq m_{1}\right\}$.

3. What is the relation between $m_{\beta}$ and $m_{\psi}$ ?

\section{Materials and Methods}

In this paper, MATLAB software and methods of mathematical analysis were used.

\section{Conclusions}

The main result of this paper is proving that the functions $Y_{m}(x)=x^{m}\left|\beta^{(m)}(x)\right|$ are not completely monotonic on $(0, \infty)$ for all $m \in \mathbb{N}$ and that the functions $x^{m-1}\left|\beta^{(m)}(x)\right|$ and $x^{m-1}\left|\psi^{(m)}(x)\right|$ are completely monotonic on $(0, \infty)$ for all $m \in \mathbb{N}, m \geq 3$.

Funding: The work was supported by VEGA grants Nos. 1/0589/17, 1/0649/17, and 1/0185/19 and by Kega grant No. 007 TnUAD-4/2017.

Acknowledgments: The author thanks to Ondrušová, dean of FPT TnUAD, and Vavro, deputy dean of FPT TnUAD, Slovakia, for their kind grant support.

Conflicts of Interest: The author declares that he has no competing interests.

\section{References}

1. Koumandos, S. Remarks on some completely monotonic functions. J. Math. Anal. Appl. 2006, 324, 1458-1461. [CrossRef]

2. Clark, W.E.; Ismail, M.E.H. Inequalities involving gamma and psi functions. Anal. Appl. 2003, 1, 129-140. [CrossRef]

3. Qi, F.; Cui, R.Q.; Chen, C.P.; Guo, B.N. Some completely monotonic functions involving polygamma functions and an application. J. Math. Anal. Appl. 2005, 310, 303-308. [CrossRef]

4. Qi, F.; Agarwal, R.P. On complete monotonicity for several classes of functions related to ratios of gamma functions. J. Ineqaul. Appl. 2019, 36, 44. [CrossRef]

5. Matejička, L. A Solution to Qi's Conjecture on a Double Inequality for a Function Involving the Tri- and Tetra-Gamma Functions. Mathematics 2019, 7, 1098. [CrossRef]

6. Matejička, L. A Solution to Fourth Qi's Conjecture on a Complete Monotonicity. Probl. Anal. Issues Anal. 2020, submitted.

7. Alzer, H.; Berg, C.; Koumandos, S. On a conjecture of Clark and Ismail. J. Approx. Theory 2005, 134, 102-113. [CrossRef]

8. Nantomah, K. Monotonicity and convexity properties of the Nielsen's $\beta$ function. Probl. Anal. Issues Anal. 2017, 6, 81-93. [CrossRef]

9. Flett, T.M. On the function $\sum_{n=1}^{\infty}(1 / n) \sin (t / n)$. J. Lond. Math. Soc. 1950, 25, 5-19. [CrossRef]

10. Gautschi, W. The Hardy-Littlewood function: An exercise in slowly convergent series. J. Comp. Appl. Math. 2005, 179, 249-254. [CrossRef]

11. Hardy, G.H.; Littlewood, J.E. Notes on the theory of series (XX): On Lambert series. Proc. Lond. Math. Soc. 1936, 41, 257-270. [CrossRef]

12. Kuznetsov, A. Asymptotic approximations to the Hardy-Littlewood function. J. Comp. Appl. Math. 2013, 237, 603-613. [CrossRef]

13. Segal, S.L. On $\sum(1 / n) \sin (x / n)$. J. Lond. Math. Soc. 1972, s2-4, 385-393. [CrossRef]

14. Alzer, H.; Berg, C. Some classes of completely monotonic functions. Ann. Acad. Sci. Fenn. 2002, 27, 445-460. [CrossRef]

15. Alzer, H. Complete monotonicity of a function related to the binomial probability. J. Math. Anal. Appl. 2018, 459. [CrossRef]

16. Boas, R.P. Signs of derivatives and analytic behavior. Am. Math. Mon. 1971, 78, 1085-1093. [CrossRef] 
17. Berg, C. Stieltjes-Pick-Bernstein-Schoenberg and Their Connection to Complete Monotonicity, Positive Definite Functions: From Schoenberg to Space.... 2008. Available online: http:/ / web.math.ku.dk//berg/ manus / castellon.pdf (accessed on 31 March 2020).

18. Boyadzhiev, K.N. Lah numbers, Laguerre polynomials of order negative one, and the nth derivative of $\exp (1=x)$. Acta Univ. Sapientiae Math. 2016, 8, 22-31. [CrossRef]

19. Demidovich, B.P. Collection of Problems and Exercises in Mathematical Analysis; Nauka: Moscow, Russia, 1990.

20. Fikhtengolts, G.M. A Course of Differential and Integral Calculus (Russian); Nauka: Moscow, Russia, 1966; Volume 2; Abbreviated version in English: The Fundamentals of Mathematical Analysis; Pergamon Press: London, UK, 1965.

21. Guo, B.N.; Qi, F. On the degree of the weighted geometric mean as a complete Bernstein function. Afr. Mater. 2015, 26, 1253-1262. [CrossRef]

22. Gao, P. Some monotonicity properties of gamma and q-gamma functions. ISRN Math. Anal. 2011, 2011, 375715. [CrossRef]

23. Gao, P. A note on the volume of sections of Bpn. J. Math. Anal. Appl. 2007, 326, 632-640. [CrossRef]

24. Qi, F.; Mahmoud, M. Completely monotonic degrees of remainders of asymptotic expansions of the digamma function. arXiv 2019, arXiv:1912.07989v1.

25. Ismail, M.E.H.; Lorch, L.; Muldoon, M.E. Completely monotonic functions associated with the gamma function and its q-analogues. J. Math. Anal. Appl. 1986, 116, 1-9. [CrossRef]

26. Koumandos, S. On completely monotonic and related functions. In Mathematics Without Boundaries; Springer: Berlin, Germany, 2014.

27. Koumandos, S. Monotonicity of some functions involving the gamma and psi functions. Math. Comp. 2008, 77, 2261-2275. [CrossRef]

28. Koumandos, S.; Lamprecht, M. Complete monotonicity and related properties of some special functions. Math. Comp. 2013, 82, 1097-1120. [CrossRef]

29. Koumandos, S.; Pedersen, H.L. Absolutely monotonic functions related to Euler's gamma function and Barnes' double and triple gamma function. Monatsh. Math. 2011, 163, 51-69. [CrossRef]

30. Koumandos, S.; Pedersen, H.L. Completely monotonic functions of positive order and asymptotic expansions of the logarithm of Barnes double gamma function and Euler's gamma function. J. Math. Anal.Appl. 2009, 355, 33-40. [CrossRef]

31. Matejíčka, L. Some notes on complete monotonicity of functions involving derivatives of the Nielsen's $\beta$-function. Probl. Anal. Issues Anal. 2020, submited.

32. Nielsen, N. Handbuch der Theorie der Gamma Funktion, 1st ed.; B.G. Teubner: Liepzig, Germany, 1906.

33. Pedersen, H.L. Completely monotonic functions related to logarithmic derivatives of entire functions. Anal. Appl. 2011, 9, 409-419. [CrossRef]

34. Roberts, A.W.; Varberg, D.E. Convex Functions; Academic Press: NewYork, NY, USA, 1973.

35. Schiff, J.L. The Laplace Transform: Theory and Applications; Springer-Verlag: New York, NY, USA; Berlin/Heilderberg, Germany, 2013.

36. Schilling, R.L.; Song, R.; Vondracek, Z. Bernstein Functions I Theory and Applications, 2nd ed.; de Gruyter Studies in Mathematics 37; Walter de Gruyter: Berlin, Germany, 2012. [CrossRef]

37. Widder, D.V. The Laplace Transform; Princeton University Press: Princeton, NJ, USA, 1946.

38. Yang, Z.H.; Zheng, S.Z. Complete monotonicity involving some ratios of gamma functions. J. Inequal. Appl. 2017. [CrossRef]

39. Zhao, J.-L. A completely monotonic function relating to the q-trigamma function. J. Math. Inequal. 2015, 9, 53-60. [CrossRef]

(C) 2020 by the authors. Licensee MDPI, Basel, Switzerland. This article is an open access article distributed under the terms and conditions of the Creative Commons Attribution (CC BY) license (http://creativecommons.org/licenses/by/4.0/). 